\title{
EMOTIONS IN SOCIAL SCIENCE: A READER ${ }^{1}$
}

\author{
EDUARD MORENO
}

\section{From a Positive Science of Emotion to Social Constructionism}

Greco and Stenner's collection represents a timely and ambitious survey of the affective turn in the social sciences. The first part of the reader reveals the importance the textual turn had for the affective turn to take place. Within the former turn, the universality of emotions was, at least, deeply challenged. The extracts in this part, mostly authored long before the affective turn took shape as such, reflect (on) the context of the textual turn in which "emotions came to be considered as discursive, dialogical phenomena, structured and influenced by the historical and cultural contingencies of communicational interactions" (p. 9). The three sections examine each of these features respectively: history, culture and society (mainly communicational interactions).

Authors such as Thomas Dixon in the first extract show how, if discourse (in the broadest sense) is not a mere representation of reality but a constitutive part of it, the different ways emotions have been conceptualized across time definitely play a role in the transformation of emotional experience, expression and its "location". Thus, aspects such as its possible relations to the human willingness are also continuously modified

Apart from this theoretical influence, shifts in the experience and expression of the affective life are intimately related to changes in social rules and standards. Both the extracts from Elias and De Swaan in this section understand the emotional and its changes as part of broader social structures and its developments. As Elias puts it, "it is the structure of society that demands and generates a specific standard of emotional control" (p. 37). To the extent that the relation between changes in emotions and the development of particular histories is reciprocal, the last excerpt of this section by Peter N. Stearns and Deborah C. Stearns shows how the influence historical changes have in shaping the emotional does not occur always at the same level nor is prompted by a single factor. This relation between history and emotion is multileveled and multifaceted.

1 Greco, Monica and Stenner, Paul: Emotions: A Social Science Reader. Oxon: Routledge 2008, 512 pp. 
In the textual turn as it is represented here, language is given a primary role in the shaping of emotions. Differences and similarities in emotional experience and expression where languages and cultural meaning systems differed attracted the attention from social sciences, especially anthropology. In the first extract Catherine A. Lutz discusses how the discourse on emotions in western societies is built around a rhetoric of control and conspicuously associated to woman, showing not only its particularity but also its political implications. Reddy, in the following section, agrees that "emotional control is the real site for the exercise of power" (p. 80) but denies its cultural specificity and reasserts the existence of an extradiscursive residuum of emotions to which language is related and influence but always fails to represent. In the third extract, the ethnohistorian of emotions Michael Harkin also argues that relying exclusively on linguistic data is not enough to understand emotional schemata and proposes to use psychodynamic approaches to give an account of ethnohistorical events as well as its previous ways of being investigated. Regardless of its nature, emotions do not only passively receive more or less cultural influence but can also be considered as a basis for theorizing social action as the final extract from Lindquist exemplifies. There, malu (meaning approximately shame, embarrassment, shyness, or restraint and property)is understood as an organizing principle for social action and the management of appearances amongst female migrants in Batam (Indonesia). Likewise, the last section of this part provides several extracts that reflect the intimate relation between emotions, society and its study. In the first extract, Barbalet relates these three aspects by associating the different approaches sociology has had towards emotions with broader social structural factors. The second extract is from Goffman's groundbreaking work that emphasized the social nature of emotions. Developments of his approach are exemplified in the following two extracts by Shott and Hochschild through the notion of social norms, which, it is claimed, highly determine emotional experiences. Kemper also focuses on the social causes of emotions in the fourth extract, albeit assuming their physiological and psychological universal stratum. . In his extract, Collins, like the previous authors, acknowledges the role of social processes in emotional formation but also emphasizes the inverse relation. To close this section and the first part, the editors include an extract from sociologist Niklas Luhman, who sidesteps the social determinism implicit in other sociological approaches by clearly distinguishing the social and psychic systems, whilst recognizing their mutual interaction and permanent tension also mirrored in the other excerpts.

To sum up, this first section reconnoitres the textual turn as a necessary reference for every attempt to review the current state of the social sciences, how they approach the affective life and what they have to offer to debates around it, namely, historical, cultural and interactional factors.

\section{From the Textual Turn to the Affective Turn in Social Science}

According to the editors, "the textual turn can be described as one of the conditions of possibility for the more recent affective turn" (p. 9). However, affect is invoked to gesture towards something that perhaps escapes or remains in excess of the practices of the speaking subject (Blackman and Venn, 2010). In sum, the affective turn is rooted in notions key to the textual turn (i.e. power, performativity and activity) but aims to extend them beyond the 
socio-cultural and include the pre-discursive (p. 10). Within this context, it is quite frequent to find how the body is brought back into theory and complicated, in Blackman and Venn's terms, by theories previously neglected by the thinkers of the textual turn since they were considered, so to speak, too "biological".

The editors devote the second part of the reader to exemplify this fact and its repercussions for numerous traditional concerns of the social sciences. Psychologist Silvan Tomkins represents a key author in the affective turn and its endeavour of offering social theory grounded in some biological accounts (p. 144). For this reason perhaps, the editors have structured the first section-on emotions, selfhood and identity-around his work. An extract of his theory of personality serves as an introduction whilst, they posit, his script theory's emphasis on the constant organization of different events is exemplified both in the extract on "coolness" by Pountain and Robins and also in Morgan and Averill's research on "true feelings". In this sense, the latter underscore that these true feelings embody a history of social meanings (p. 164). It is precisely this relation between emotions and the normative characteristics of a given personhood such as that characteristic of Western societies which is analysed in the third extract. There, Agneta H. Fischer and Jeroen Jansz argue that "emotions are not absent from Western personhood and that our cultural ideal of a person requires a form of bounded emotionality" (p. 173). It is also worth noting how the affect of "shame" occupies a central position not only in Tomkins' theorization (see Sedgwick and Frank 1995) but also in many social theorists concerned with the self, such as W. Ray Crozier, in the closing extract of this section.

In the second section, it is emphasised how body-studies are complicated within the affective turn and the materialization of affect goes beyond the body. Less attention is paid to the relation between the self and the (inner) emotions. Actually, with theorists such as Thrift there is argued to be a rather too hasty dismissal of the concept of subjectivity (Stenner 2008). In the first extract, Thrift himself shows how the systematic engineering of affect is now central to the political life of Euro-American cities. The extent to which these "affectively engineered" spaces are subsequently felt in particular ways is exemplified in the second and third extracts, by Rani Kawale and Mimi Sheller respectively. The former explains that "spaces are not necessarily sexualised only by physical behaviour, but by the reading of emotions attached to those bodily acts by onlookers" (p. 191), whilst the later pays attention to the emotional constituents of car cultures, shifting away from the rational actor (p. 194). In the final extract, Ben Anderson explores the role of affect in everyday judgements and how these are "not based on an already decided preference, or taste, but instead is bodied-forth without deliberation from within the immediacy of a given situation (Varela 1999, 9)" (p. 202).

Inasmuch as the body is also a key concern in research on health and illness, the role emotions play in this field is explored in the last section of this part devoted to "embodied affects". Healthcare practices and places have been frequently depicted as sites where positivism (or the crude body) and social constructionism (or society) clearly collide. However, according to Peter E. Freund (in the first extract of this section), the body is a means of expressing meaning, including socio-cultural meanings (p. 212). Consequently, that relation of opposition must be rethought, as the other extracts in this section exemplify. The extract by Alan Radley is an illustration of how "medical humanities" can complement 
biomedical "objectivism" by examining how to comprehend another's suffering is grounded on the observer's emotional responses. The following text from Wilce and Price offers "a form of critique designed to feed into and improve (rather than deconstruct or contextualize) psychoneuroimmunological hypothess on the role of emotions in health and illness" (p. 207). Still against the traditional antibiologism characteristic of contemporary social theory, Elizabeth A. Wilson's extract is "governed by (her) conviction that effective political engagement with the contemporary life sciences requires ongoing intimacy with their data" (p. 244). Nikolas Rose, in the final extract of this section devoted to emotions and health, gives an account of the growth of mental illness that goes beyond the traditional psychosocial factors such as poor social circumstances and includes some characteristics from the psychiatric science and practice itself as significant causes.

Whilst the textual turn definitely changed the social sciences by introducing new theories, methodologies and themes such as emotions, some of its interpretation left the body out of their concerns. However, this recovered interest in emotions allowed the affective turn to take form as such precisely by seriously trying to overcome this elusion. The second section of the reader gives a complete account of this endeavour, how emotions are currently conceived and are also changing the social sciences scene itself.

\section{Disciplining Affects}

The third and fourth parts of the reader are used to outline how the link between affective life and relations of power is a clear common denominator in research about affect in social sciences (p. 12). The normative dimensions of knowledge about emotion become a central concern in this new field. Therefore, how this knowledge is built is critically addressed throughout the first section of the third part. Methodological issues and its consequences in the context of work and organization are treated with a deeper detail in all the extracts of this section. Kiran Mirchandani, in the first extract, exposes the normalization of whiteness implicit in contemporary debates on emotion work, mainly as a result of methodological decisions. The second extract, by Stephen Fineman, shows the disciplining consequences of constructing and measuring emotional intelligence in organizational environments. A different use of quantitative research is exemplified in the text by Reeve et al. In particular, it serves to investigate "the relatively neglected dimension of emotion in situations of interaction across species" (p. 262). The last extract in this section, by Smith and Kleinman, goes back to a medical context to explore how emotions can also be apparently and actively ignored in given environments but implicitly socialized.

It is perhaps the extracts in the second section which most pointedly serve a broader purpose than merely representing research on emotion, economics and consumer culture. Taken together these texts make a strong case that the boundary between emotion and reason is no longer an obvious one and does not represent a relation of opposition. The rational and self-interested actor traditionally assumed by classical accounts of economic theory is argued to be embedded in affect or, as the editors put it referring the last extract of this section, "emotions can shape the very ability to make rational choices" (p. 300). According to Campbell, romanticism is not only material used and constructed by advertisers (as the extract by Lefkowitz in this section shows) but it also "played a critical role in facilitating 
the Industrial Revolution and therefore the character of modern economy" (p. 302). Danny Miller, in the second extract, argues that everyday shopping can be seen as "making love", the later understood as a "normative ideology manifested largely as a practice within longterm relationships" (p. 309).

According to the editors, probably the main mediator in these processes of change and reproduction is the media which "have taken over the socializing functions previously performed by the family" (p. 321). It is, for instance, capable of shaping not only our affective experiences as Höijer exemplifies in the first extract but also, by means of them amongst other things, what Tayler in the second extract refers to as "figures" (comparable to Moscovici's Social Representations). On the other hand, given the prominence of authenticity in our current societies, an industry exists around it and its core element (emotions) of which reality TV is an important actor, as Aslama and Pantti show in the third text of this section. However, emotions are not only subjects of which people talk about in the media. Smith argues, in the final extract, that "generating brief, intense emotions often requires an orienting state (a mood) created by certain film structures" (p. 347).

It is possible to perceive how the third part of the reader leaves behind how the affective turn was defined and focuses mostly on its repercussions on the social sciences. The extracts in this section show how their methods, theories and practical applications are still being deeply challenged and transformed in some cases.

As the editors put it, "once emotions are acknowledged as not compatible with, but as an active ingredient of rational action, they also become recognizable as a central factor in political analysis" (p. 357). These are the kind of texts gathered in the fourth part, conspicuously in the first section. Reflection on feelings is increasingly present in political planning and communication, as Barry Richards' excerpt denotes. Fortier argues in the second extract that, once they start to circulate, emotions are also increasingly a core element "in policing the terms of belonging and entitlement to citizenry" (p. 368). Therefore, emotions can easily become a "technology of governance" (p. 359) an example of which is hope, as outlined in the extract by Shearing and Kempa. Finally, despite being sometimes intentionally and institutionally articulated, Deborah Gould draws on Reddy's notion of emotive to understand emotional experience also as a "reservoir of possibilities of change" (p. 359).

By dealing with the legal system as a key aspect of politics, the section on emotions and law offers opportunities to clearly observe the relation between emotions and morality. According to Laster and O'Malley's opening extract, "the incorporation of the emotional into law and legal rhetoric is manifest in a wide array of areas of law" such as legal definitions, judge's instructions, new civil harms, etc. Given their involvement in the creation of law simultaneous to their presence in a private sphere of civil society, emotions are conceived as a "link concept" between these two realms by Bettina Lange in the second extract. Accordingly, Arie Freiberg argues that crime prevention strategies are more likely to be successful if they recognize and deals with the affective. The fourth extract in this section, by Navarro-Yashin, examines the role of material aspects of law such as documents in these emotional dynamics. This broad "emotionalization of law" is critically addressed in the last extract of this section by Susanne Karstedt.

The closing section of the book, dedicated to the emotions of compassion, hate and terror, shows how they sometimes act as "unifying principles" (p. 425). This can be found 
especially in the three intermediate texts. According to the editors, Berlant suggest in his extract that compassion might be better thought as a "social and aesthetic technology of belonging rather than some authentic, organic emotion". Secondly, the extract by Judith Butler explores whether a "basis for community" can be found in our ways of mourning and our sense of vulnerability. Hatfield and Rapson engage with the controversial notion of "emotional contagion" in order to "provide a few insights into the powerful forces that unite people and divide them from their fellows" (p. 443). The first and last extracts reveal respectively how "language itself becomes a painful and fraught minefield of confusion" (p. 426) and "compassion (is) an organized effort to lessen the suffering of strangers" in modern societies (p. 427).

The last part of the reader gives examples of how the turn to emotions, by affecting social sciences, also allows them not only to reinvent themselves but also to find new ways of understanding and reporting political actions and effects. In addition to this vast array of texts and authors, the book finishes with a helpful and comprehensive guide for further reading that strengthens its position as an "obligatory passage point" for students and social scientists interested in emotions and involved the affective turn.

\section{Transcendent Themes and Valorisation}

The editors draw almost exclusively from qualitative research, largely because it has been central to the challenge to "the scientific superiority of detached reason and objective observation over the emotional and the subjective" (p. 6). However, this primacy is also confronted within the affective turn by, for instance, reengaging with the body and its different ways of studying it. As the editors put it, "despite a reconnection with issues of materiality and embodiment, what is involved in the affective turn is by no means a return to na ve representationalism associated with positivism" (p. 9-10) and this is why, conspicuously in the section devoted to emotions in work and organizations, room for certain uses of quantitative research is found. Nevertheless, conventional oppositions such as this between qualitative and quantitative research are currently being challenged within the affective turn (Athanasiou et al. 2008) and requires a creative and innovative development of method (Blackman and Venn 2010). Perhaps due to its publication date and its aim to trace the whole turn to affect, these new ideas are still not reflected in the reader.

Regarding the editor's presence in the reader, some might prefer extended extracts instead of the introductions or a shorter selection of excerpts, since the exercise of pointing at aspects of mutual relevance can make some pieces appear repetitive. However it would be worth insisting on the appropriateness of the general and each part introductions. They compensate admirably for the inevitable edition and compression of the extracts. Whilst on the surface, this book does the job of a standard reader in that it is a collection of representative texts regarding the affective turn, on closer reading, it achieves something more than the sum of its parts. Via the selection of texts and the editor's narrative, it manages to capture "the more general significance of the study of affect and emotions in social science" (p. 2) as encapsulated in the affective turn. It attunes the reader to the conviction that "an engagement with affective life has the potential to transform the ways in which social science disciplines conceive their own way of knowing and their objects of research" (p. 5). The introductions 
effectively frame the four sections of the book as elements of a coherent project instead of isolated areas of knowledge, which is a significant achievement. To sum up, the reader offers not only a pertinent "moving" picture of the state of the field of social studies of emotion but also, making the most of the complexity involved in having to select texts and authors from an immense array of fields and perspectives, contributes to the development of the affective turn by signalling its most promising points, its redundancies, weakness and strengths.

\title{
References
}

Athanasiou, A., Hantzaroula, P., Yannakopoulos, K. Towards a New Epistemology: The "Affective Turn”. Historein 8, 5-16, 2008.

Blackman, L., Venn, C. Affect. Body \& Society 16, 7-28, 2010.

Stenner, P. A. N. Whitehead and Subjectivity. Subjectivity 22, 90-109, 2008.

\author{
University of Brighton \\ Applied Social Science \\ Mayfield House \\ Falmer \\ Brighton (United Kingdom) \\ BN1 9PH
}

Tel.: 0034679366022

E-mail: 160@brighton.ac.uk 